Artículo científico

Volumen 32(1):63-76. Enero-abril, 2021

e-ISSN 2215-3608, doi:10.15517/am.v32i1.39035

http://www.revistas.ucr.ac.cr/index.php/agromeso

\title{
Análisis físico-químico de filetes de la especie exótica Hemiancistrus aspidolepis en Costa Rica ${ }^{1}$
}

\section{Physico-chemical analysis of fillets of the exotic species Hemiancistrus aspidolepis in Costa Rica}

\author{
Nelson Peña-Navarro², Paulina Vargas-Alpízar ${ }^{3}$
}

1 Recepción: 5 de febrero, 2020. Aceptación: 21 de septiembre, 2020. Este trabajo se realizó como parte de los objetivos del proyecto Conservación, uso sostenible de la biodiversiddad y mantenimiento de los servicios de los ecosistemas de humedal de importancia internacional (Proyecto Humedales), Programa de las Naciones Unidas para el Desarrollo - Sistema Nacional de Áreas de Conservación, $2014-2019$.

2 Universidad Técnica Nacional, Dirección de Investigación, Sede del Pacífico, Puntarenas, Costa Rica. npena@utn.ac.cr (autor para correspondencia, https://orcid.org/0000-0002-8916-812X).

3 Programa de las Naciones Unidas para el Desarrollo - Sistema Nacional de Áreas de Conservación, Proyecto humedales, San José, Costa Rica.pvargasalpizar@gmail.com (https://orcid.org/0000-0003-1850-3004).

\section{Resumen}

Introducción. Cuando especies de animales invasoras se introducen en un ecosistema que no es su área de distribución natural, su control podría estar determinado por la posibilidad de utilizar estos organismos para el consumo humano u otra alternativa de manejo. Objetivo. Determinar las características físico-químicas del filete de H. aspidolepis, y comparar su calidad nutricional con especies de peces de consumo humano de origen marino y de aguas continentales. Materiales y métodos. Se recolectaron 109 peces en tres Refugios de Vida Silvestre en Costa Rica, durante el 2017, se pesaron en fresco y sin vísceras, además se pesó cada filete, las vísceras y la carcasa. Se les realizó un análisis proximal, para determinar el contenido de ácidos grasos, minerales y mercurio. Resultados. La longitud promedio de los individuos recolectados fue de $25,35 \mathrm{~cm}$ y el peso promedio fue de $162,55 \mathrm{~g}$, de los cuales el 8,2 \% fueron vísceras, y 19,02\% fue músculo. Para cada muestra se obtuvieron promedios por cada $100 \mathrm{~g}$ de: $80,69 \mathrm{~g}$ de agua, $17,64 \mathrm{~g}$ de proteína y 73,40 kcal de energía. Los niveles de grasa trans y carbohidratos totales fueron

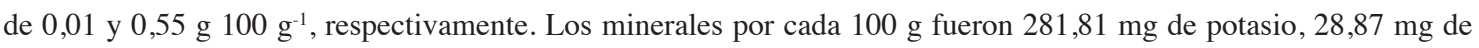
sodio y 1,23 mg de hierro. Los ácidos grasos en mayor cantidad fueron: palmítico $(23,64 \%)$, esteárico $(9,43 \%)$ y tricosanoico $(7,26 \%)$. El nivel de mercurio total fue inferior a 0,15 ppm. Conclusiones. Los individuos colectados no poseían tallas ni porcentajes de peso de filetes similares a los utilizados para mercado y consumo humano. $H$. aspidolepis posee semejanzas en contenido proximal, ácidos grasos y minerales con respecto a algunos peces de consumo humano; los contenidos de proteínas y lípidos la hacen apta para su consumo y comercialización.

Palabras clave: análisis proximal, seguridad alimentaria, pez silvestre, proteína animal.

\begin{abstract}
Introduction. When invasive animal species are introduced into an ecosystem that is not their natural range, their control could be determined by the possibility of using these organisms for human consumption or other management
\end{abstract}


alternatives. Objective. To determine the physical-chemical characteristics of the H.aspidolepis fillet, and to compare its nutritional quality with fish species for human consumption of marine origin and continental waters. Materials and methods. 109 fish were collected in three Wildlife Refuges in Costa Rica, during 2017, they were weighed fresh and without viscera, in addition each fillet, viscera, and carcass were weighed. A proximal analysis was performed to determine the content of fatty acids, minerals, and mercury. Results. The average length of the collected individuals was $25.35 \mathrm{~cm}$ and the average weight was $162.55 \mathrm{~g}$, of which $8.2 \%$ were viscera, and $19.02 \%$ were muscle. For each sample, there were obtained averages per $100 \mathrm{~g}$ of: $80.69 \mathrm{~g}$ of water, $17.64 \mathrm{~g}$ of protein and $73.40 \mathrm{kcal}$ of

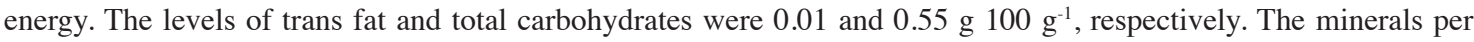
$100 \mathrm{~g}$ were $281.81 \mathrm{mg}$ of potassium, $28.87 \mathrm{mg}$ of sodium, and $1.23 \mathrm{mg}$ of iron. The fatty acids in greater quantity were: palmitic $(23.64 \%)$, stearic $(9.43 \%)$, and tricosanoic $(7.26 \%)$. The total mercury level was less than 0.15 ppm. Conclusions. The collected individuals did not have similar sizes and weight percentages of fillets to those used for market and human consumption. H. aspidolepis has similarities in proximal content, fatty acids, and minerals with respect to some fish for human consumption; the contents of proteins and lipids make it suitable for consumption and commercialization.

Keywords: proximal analysis, food security, wild fish, animal protein.

\section{Introducción}

Las especies invasoras son aquellas que se han introducido en un ecosistema que no es su área de distribución natural y donde logran colonizar y establecerse (Aguirre-Muñoz et al., 2009) al superar barreras geográficas, ambientales, de cautiverio, reproductivas, entre otras (Blackburn et al., 2011). Además, una especie introducida se convierte en invasora al causar deterioro ambiental o económico al ser humano (Deines, 2013). En los ecosistemas de humedal las oportunidades para las invasiones son amplias, debido a que la gama de hábitat es muy variada, van de habitats terrestres a acuáticos incluidos los ecosistemas marinos, además de la gran probabilidad de que estos cambien (Howard, 1999).

En Costa Rica, la tilapia (Oreochromis noliticus), la trucha arcoíris (Oncorhynchus mykiss) y la carpa común (Cyprinus carpio), fueron introducidas para el desarrollo de la acuicultura para consumo humano, mientras que otras especies como la carpa dorada (Carassius auratus), la olomina (Poecilia reticulata), el platí (Xiphophorus variatus) y el pez pleco leopardo (Pterygoplichthys pardalis) se introdujeron como especies ornamentales, muchas de las cuales son actualmente consideradas por la población como especies exóticas invasoras (Deines, 2013; Guerrero, 2016; Castillo, 2018).

La especie del pez diablo o pleco Hemiancistrus aspidolepis (Angulo et al., 2013), es una de las dos especies de plecos del sur del país, la cual se reporta distribuida en la vertiente Pacífica entre los ríos Grande de Térraba de Costa Rica y el río Tuyra de Panamá (Bussing, 2002). Sin embargo, actualmente se le encuentra en ríos y humedales del Caribe y Norte del país, como los ríos: Frío, San Carlos, Sarapiquí, Colorado y San Juan.

H. aspidolepis, pertenece a la familia Loricariidae, familia originaria de Sudamérica que se caracteriza por tener una coraza de placas óseas que cubre todo el cuerpo y cabeza, excepto el vientre (Bussing, 2002), posee una dieta básica de detritus, algas y algunos invertebrados bentónicos (Covain \& Fisch-Muller, 2007). Alcanzan longitudes de hasta los $80 \mathrm{~cm}$ (Hoover et al., 2014) y su madurez sexual llega a los 12,5-13,0 cm de longitud, necesitan elaborar cavidades en las paredes de ríos y lagunas para anidar y colocar sus huevos (Gibbs et al., 2008; Nico et al., 2009; Hoover et al., 2014). 
Cuando se determinó la abundancia de la especie, las relaciones longitud-peso y los aspectos reproductivos de H. aspidolepis en la vertiente Atlántica norte de Costa Rica, se concluyó que este pez es la especie capturada con mayor abundancia y biomasa total, posee procesos reproductivos establecidos, por lo que su invasión es de forma extendida y dominante (Castillo, 2018). Algunos estudios sugieren la posibilidad de utilizar especies consideradas como invasoras para el consumo humano, como ha sido el caso de la tilapia en Costa Rica, cuya presencia se ha controlado en los ríos y lagunas, debido a que es consumida por los pobladores y esto ha generado una reducción de su impacto ambiental (Castillo, 2018). H. aspidolepis y otras especies de este género no afectan la salud de las personas, pueden ser comestibles y obtenerse subproductos para uso humano y animal (Castillo, 2018). Además, se debe considerar que los pescados poseen un aporte valioso como alimento en la dieta humana por su contenido nutricional y su relación entre su consumo y un adecuado estado de salud (Fonseca-Rodríguez \& Chavarría-Solera, 2017), influenciado por su alto contenido de ácidos grasos poliinsaturados (Njinkoue et al., 2016).

H. aspidolepis, se considera un pez magro debido a su bajo contenido de grasa que es inferior al $3 \%$ (Varlik et al., 2004) y en comparación a otras especies de peces que tienden a poseer mayores contenidos grasos como el caso de la tilapia, el pargo y la corvina (Izquierdo-Córser et al., 2000) o el lenguado, la barracuda y la anguila (Fonseca-Rodríguez \& Chavarría-Solera, 2017).

Al analizar alternativas para el control de H. aspidolepis en el humedal Ramsar de Caño Negro, se determinó la factibilidad de implementar la creación de harina de pescado a partir de este pez, como alternativa para mitigar su impacto negativo y a la vez mejorar la condición socio-económica de la zona (Fonseca \& Vargas, 2018). Por lo consecuente, para organismos introducidos, nuevos en un ecosistema y poco conocidos por la población, se hace importante realizar estudios de estos animales como fuente de proteínas, ácidos grasos y minerales, con la finalidad de analizar si son adecuados para el consumo humano (Izquierdo-Córser et al., 2000) y si lo son, qué aporte nutricional estarían brindando. El consumo de alimentos con una mayor cantidad de ácidos grasos insaturados (AGI) se relaciona con una mejor condición médica asociada a la disminución de accidentes cardiovasculares (Carrillo et al., 2011), y el consumo de peces bajos en ácidos grasos saturados (AGS) y altos en ácidos grasos poliinsaturados (AGPI), mejoran la salud de los seres humanos cuando su consumo es constante (Undeland et al., 2004), con beneficios en la reducción de inflación crónica, depresión, lesiones cardiacas, entre otras (Castro et al., 2013).

Dado que algunos de estos humedales son también sitios Ramsar, el pez pleco ha sido objeto de estudio por la Unidad Técnica del Proyecto Humedales PNUD-SINAC (Proyecto Humedales de Sistema Nacional de Áreas de Conservación - Programa de Naciones Unidas para el Desarrollo, Global Enviromental Facility, 2018), en respuesta a la Resolución VIII.18. de Ramsar, la cual insta a hacerse cargo de los problemas que plantean las especies invasoras de peces en ecosistemas de humedales. El objetivo del presente estudio fue determinar las características físico-químicas del filete de H. aspidolepis, y comparar su calidad con otras especies de peces de consumo humano de origen marino y de aguas continentales.

\section{Materiales y métodos}

\section{Muestreo de organismos}

Los pescados de $H$. aspidolepis evaluados en este trabajo fueron capturados en los Refugios Mixtos de Vida Silvestre y Sitios Ramsar: Caño Negro (Laguna Caño Negro y San Sebastián), Maquenque (Río San Carlos a la altura de Boca Tapada) y Barra del Colorado (Isla Brava y Puerto Lindo), durante el primer semestre de 2017 (Figura 1), como parte de los muestreos realizados por el Proyecto Humedales para obtener información de línea base de la ecología de esta especie de Loricaridos. 

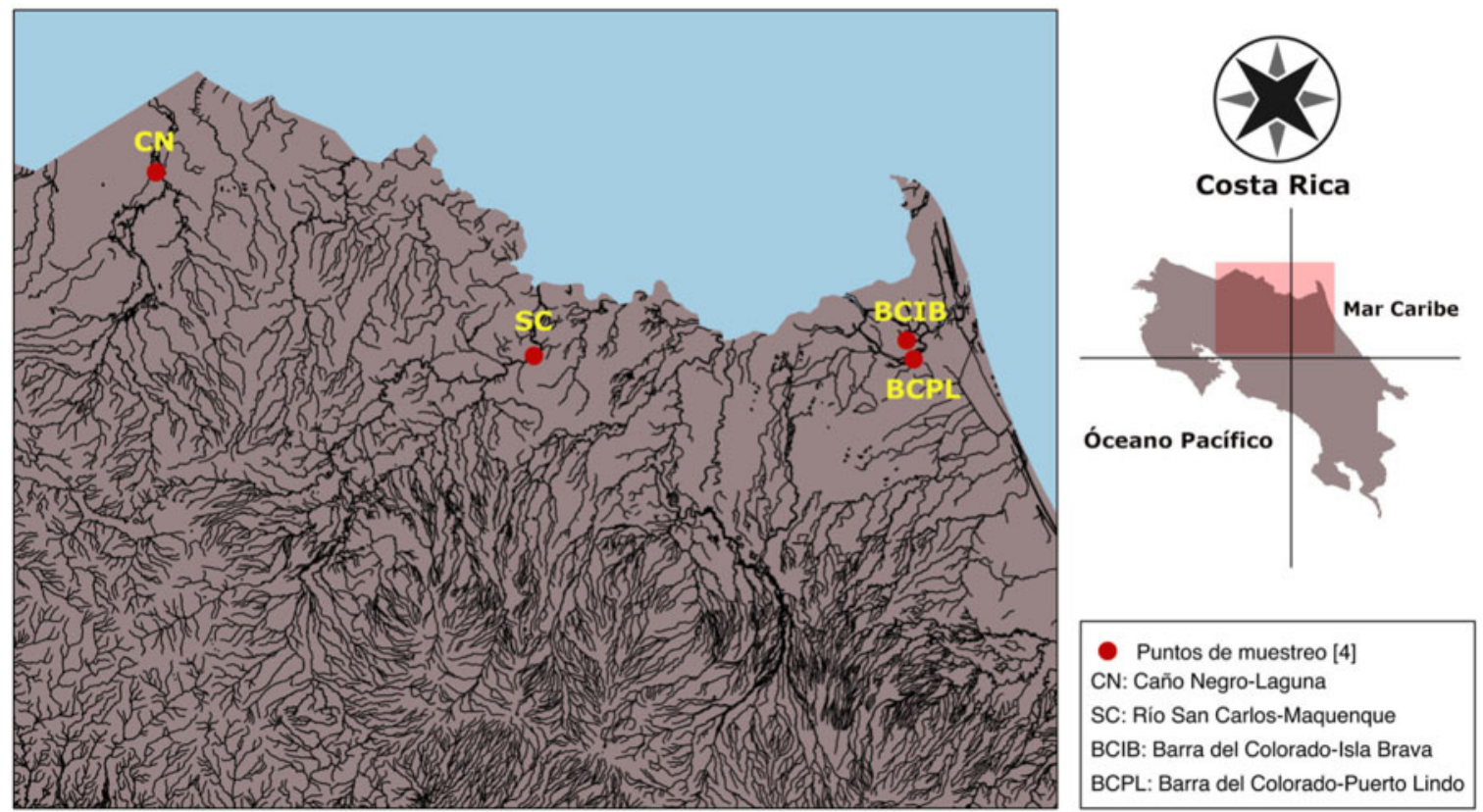

Figura 1. Sitios de colecta de Hemiancistrus aspidolepis en Refugios Mixtos de Vida Silvestre en Costa Rica. 2017.

Figure 1. Hemiancistrus aspidolepis sampling sites in Mixed Wildlife Refuges in Costa Rica. 2017.

Se recolectaron 109 organismos, para lo que se utilizó una atarraya de filamento de nilón con 2,9 m de apertura y una luz de malla de $3 / 4$ de pulgada. Para cada punto de muestreo se realizaron cincuenta lances, definidos como buenos cuando la apertura fue mayor a $70 \%$ del área de la atarraya y que cayera sin obstrucción de troncos u otros objetos. Todos los ejemplares capturados fueron preservados para su análisis mediante congelamiento (-10 $\left.{ }^{\circ} \mathrm{C}\right)$ y trasportados en hieleras a la Estación Biológica del Parque Ecológico del Sistema Nacional de Áreas de Conservación de Costa Rica (SINAC).

\section{Caracterización física de las muestras}

Cada uno de los peces analizados fue pesado de forma completa en fresco y luego eviscerado, además, se obtuvo el peso de cada filete, las vísceras y la canal, utilizando una balanza digital de $\pm 0,001 \mathrm{~g}$ de presición. Los pescados fueron medidos por su longitud total $(\mathrm{cm})$, abarcando desde la sínfisis mandibular hasta la extremidad distal del rayo más largo de la aleta caudal ventral, con el lóbulo extendido normalmente. Se revisaron los individuos internamente y externamente con la finalidad de inspeccionar si las hembras tenían huevos y si presentaban algún endo o ecto parásito.

\section{Obtención del filet de $\boldsymbol{H}$. aspidolepis}

Se obtuvieron dos filetes enteros para cada uno de los pescados con un cuchillo afilado. Se realizó un corte longitudinal a lo largo de la espina, la incisión se hizo detrás de la cabeza y hasta el espinazo (Figura 2). 


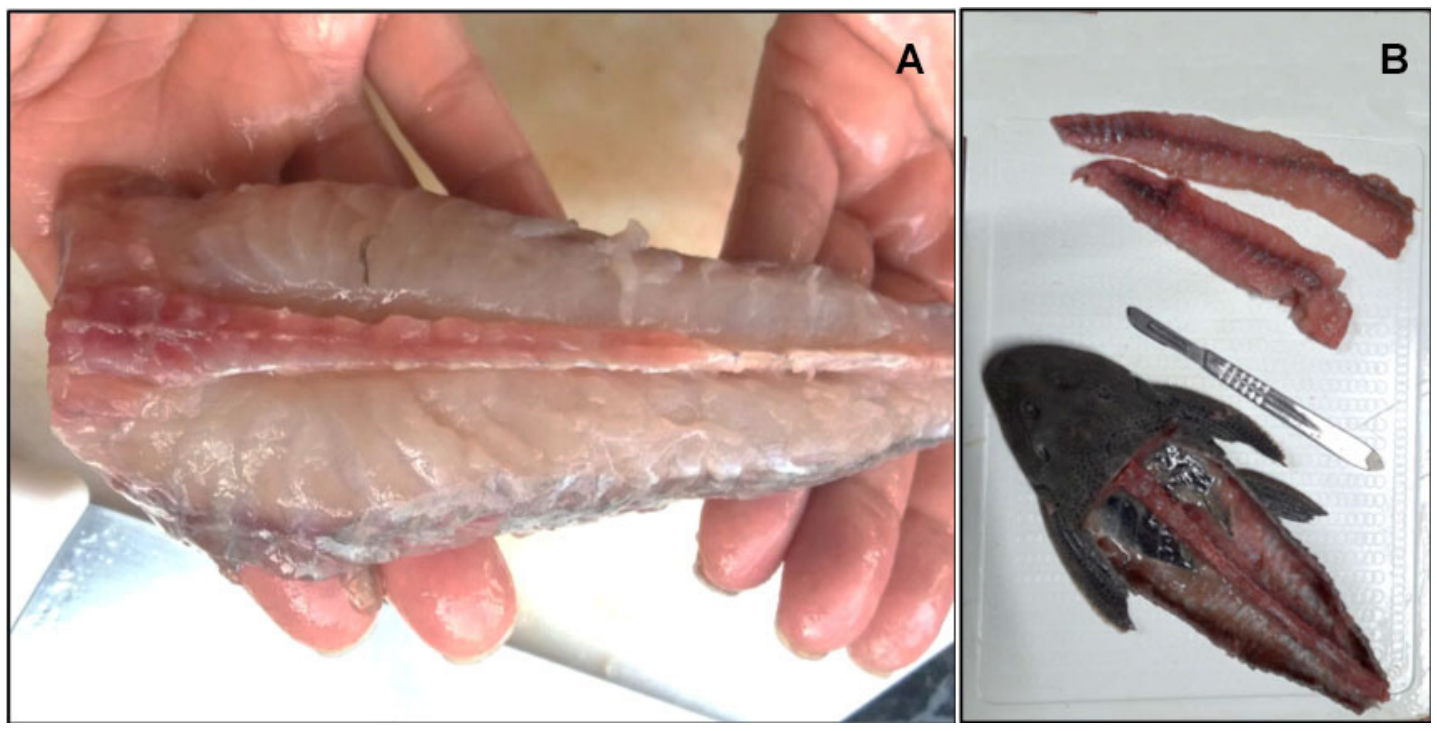

Figura 2. A) Corte longitudinal del lomo de Hemiancistrus aspidolepis, para mostrar el músculo. B) Espécimen de Hemiancistrus aspidolepis con sus dos filetes enteros. Costa Rica, 2017.

Figure 2. A) Longitudinal cut of the Hemiancistrus aspidolepis loin, to show the muscle. B) Hemiancistrus aspidolepis specimen with its two whole fillets. Costa Rica, 2017.

\section{Caracterización química de los filetes}

Se obtuvieron conjuntos de músculos de diferentes ejemplares, ya que por el tamaño de los animales se necesitaron filetes de varios individuos para conformar una unidad de $150 \mathrm{~g}$ y hasta obtener diez submuestras compuestas, las cuales fueron transportadas en contenedores cubiertos con hielo, al Centro Nacional de Ciencia y Tecnología de Alimentos (CITA) de la Universidad de Costa Rica.

A cada submuestra compuesta se le realizó el análisis proximal según los métodos recomendados por la Sociedad Oficial de Química Análitica (AOAC, por sus siglas en inglés): húmedad $(\mathrm{H})$ por secado a $110^{\circ} \mathrm{C}$ en una estufa durante 24 h, proteínas $(\mathrm{P})(\mathrm{Nx} 6,25)$ por el método de Kjeldahl, cenizas $(\mathrm{C})$ por combustión a $550^{\circ} \mathrm{C}$ durante $12 \mathrm{~h}$ en una mufla y grasa $(\mathrm{G})$ por el método gravimétrico. Además, carbohidratos totales $(\mathrm{CH})(100-\% \mathrm{H}-\% \mathrm{C} . \% \mathrm{P}-$ $\% \mathrm{G})$, valor energético en $\mathrm{kcal}(4 \mathrm{x} \% \mathrm{CH}$ disponible+4x\% $\mathrm{P}+9 \mathrm{x} \% \mathrm{G}))$ y valor energético por grasa en $\mathrm{kcal}(9 \mathrm{x} \% \mathrm{G})$ (Association of Official Analytical Chemists, 2019; Izquierdo-Córser et al., 2000).

La determinación del perfil de ácidos grasos se realizó aplicando los protocolos de la AOAC, métodos 948.15, 996.06 basado en cromatografía de gases y para minerales el protocolo AOAC, métodos 985.35 y 999.11, basado en espectrofotometría de absorción atómica (Association of Official Analytical Chemists, 2019). Se determinó el contenido de mercurio total (Hg-T), mediante la técnica de vaporización térmica (DMA) (Ruíz, 2016). Para cada muestra de músculo se tomó aproximadamente $150 \mathrm{mg}$ para su respectivo análisis. Todas las muestras se analizaron por duplicado.

\section{Análisis estadístico}

Los datos se presentan como media \pm desviación estándar. Se realizó una comparación de las medias obtenidas de las submuestras compuestas de H. aspidolepis con las medias obtenidas en la literatura, donde realizaron los 
mismos procedimientos con peces de origen marino y de aguas continentales. El análisis estadístico se realizó con el paquete estadístico SPSS ${ }^{\circledR}$. Se utilizó el análisis de varianza para detectar si existieron diferencias entre las medias y el procedimiento de Duncan para comparar las medias.

\section{Resultados}

\section{Caracterización física}

El peso promedio de los individuos colectados fue de 162,55 g, de los cuales el 8,2 \% fueron vísceras, 19,02 $\%$ fue músculo, traducido en dos filetes y el resto comprendió la carcasa donde se incluía la cabeza y cualquier otro tejido adicional (Cuadro 1).

Cuadro 1. Caracterización física de Hemiancistrus aspidolepis colectados en refugios mixtos de vida silvestre en Costa Rica. 2017.

Table 1. Physical characterization of Hemiancistrus aspidolepis sampled in Mixed Wildlife Refuges in Costa Rica. 2017.

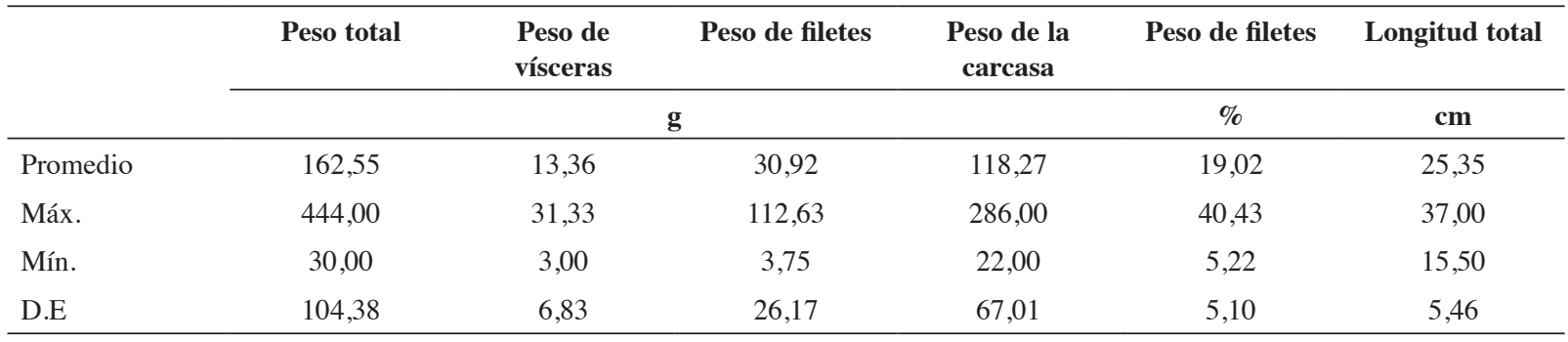

Máx: máximo; Mín: mínimo; D.E: desviación estándar / Máx: maximum; Min: minimum; D.E.: standard deviation.

El 15,6 \% (17/109) fueron especímenes que se encontraron entre los 300 y $400 \mathrm{~g}$, y solo 1,8 \% (2/109) pesaron más de 400 g. La longitud de los animales no superó los $37 \mathrm{~cm}$, y los más pequeños apenas superaron los 15 $\mathrm{cm}$. No se encontraron endo ni ectoparásitos y solo se presentaron cinco especímenes con masa de huevos en la cavidad torácica, por lo que se presume que para las fechas de colecta, la población se encontraba iniciando la fase reproductiva.

\section{Análisis proximal y valor energético}

El agua fue el componente de mayor presencia en los filetes de los pescados, teniendo 80,69 $\mathrm{g}$ en cada $100 \mathrm{~g}$.

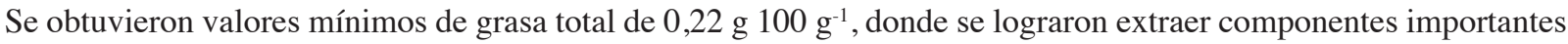
de ácidos grasos saturados, insaturados y poliinsaturados. Los niveles de grasa trans y carbohidratos totales fue

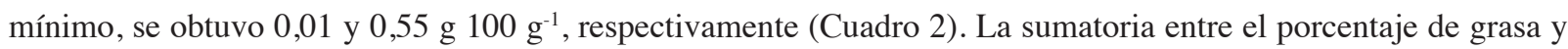
humedad fue aproximadamente de $81 \%$.

La proteína constituyó un 17,64 \%, lo que representa un valor energético de 73,40 kcal por cada $100 \mathrm{~g}$ de filete. El índice de jugosidad, traducido en la relación humedad/proteína (H/P) fue de 4,57. 
Cuadro 2. Análisis proximal y valor energético de filetes de Hemiancistrus aspidolepis colectados en refugios mixtos de vida silvestre en Costa Rica. 2017.

Table 2. Proximal analysis and energy value of Hemiancistrus aspidolepis fillets sampled in Mixed Wildlife Refuges in Costa Rica. 2017.

\begin{tabular}{|c|c|c|c|c|}
\hline & Promedio & Máx. & Mín. & D.E \\
\hline Humedad (g $\left.100 \mathrm{~g}^{-1}\right)$ & 80,69 & 81,50 & 79,76 & 0,64 \\
\hline Cenizas (g $100 \mathrm{~g}^{-1}$ ) & 1,04 & 1,21 & 0,95 & 0,08 \\
\hline Grasa $\left(\mathrm{g} 100 \mathrm{~g}^{-1}\right)$ & 0,22 & 1,12 & 0,10 & 0,32 \\
\hline Grasa saturada $\left(\mathrm{g} 100 \mathrm{~g}^{-1}\right)$ & 0,04 & 0,09 & 0,02 & 0,02 \\
\hline Grasa monoinsaturada $\left(\mathrm{g} 100 \mathrm{~g}^{-1}\right)$ & 0,04 & 0,08 & 0,02 & 0,02 \\
\hline Grasa poliinsaturada $\left(\mathrm{g} 100 \mathrm{~g}^{-1}\right)$ & 0,03 & 0,04 & 0,02 & 0,01 \\
\hline Grasa poliinsaturada (g $\left.100 \mathrm{~g}^{-1}\right)($ Omega-3) & 0,00 & 0,01 & 0,00 & 0,00 \\
\hline Grasa poliinsaturada (g $\left.100 \mathrm{~g}^{-1}\right)($ Omega-6) & 0,03 & 0,03 & 0,02 & 0,00 \\
\hline Grasa trans $\left(\mathrm{g} 100 \mathrm{~g}^{-1}\right)$ & 0,01 & 0,05 & 0,00 & 0,02 \\
\hline Proteína (g $100 \mathrm{~g}^{-1}$ ) & 17,64 & 18,92 & 16,02 & 0,93 \\
\hline Carbohidratos totales $\left(\mathrm{g} 100 \mathrm{~g}^{-1}\right)$ & 0,55 & 2,30 & 0,00 & 0,70 \\
\hline Valor energético (kcal $100 \mathrm{~g}^{-1}$ ) & 73,40 & 77,00 & 70,00 & 2,50 \\
\hline Valor energética por grasa ( $\left.\mathrm{kcal} 100 \mathrm{~g}^{-1}\right)$ & 0,60 & 2,00 & 0,00 & 0,70 \\
\hline
\end{tabular}

Máx: máximo; Mín: mínimo; D.E: desviación estándar/ Máx: maximum; Min: minimum; D.E.: standard deviation.

\section{Contenido de minerales}

Se analizaron cuatro minerales de importancia en la dieta de los seres humanos. El mineral que se presentó

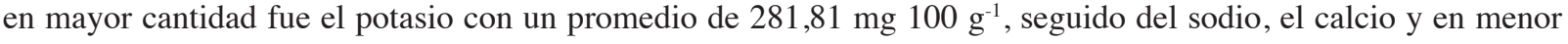
cantidad el hierro (Cuadro 3).

Cuadro 3. Contenido mineral de filetes de Hemiancistrus aspidolepis colectados en refugios mixtos de vida silvestre en Costa Rica. 2017.

Table 3. Mineral content of Hemiancistrus aspidolepis fillets sampled in Mixed Wildlife Refuges in Costa Rica. 2017.

\begin{tabular}{|c|c|c|c|c|}
\hline & Promedio & Máx. & Mín. & D.E \\
\hline & \multicolumn{4}{|c|}{$\operatorname{mg} 100 \mathrm{~g}^{-1}$} \\
\hline Sodio $(\mathrm{Na})$ & 80,99 & 133,65 & 62,40 & 22,28 \\
\hline Calcio $(\mathrm{Ca})$ & 28,87 & 47,10 & 9,18 & 13,05 \\
\hline Potasio $(\mathrm{K})$ & 281,81 & 598,67 & 186,08 & 118,95 \\
\hline Hierro $(\mathrm{Fe})$ & 1,23 & 2,86 & 0,80 & 0,63 \\
\hline
\end{tabular}

Máx: máximo; Mín: mínimo; D.E: desviación estándar. / Máx: maximum; Min: minimum; D.E.: standard deviation. 


\section{Contenido y tipos de ácidos grasos}

Se presentó el contenido de nueve ácidos grasos saturados (AGS), diez ácidos grasos monoinsaturados (AGMI) y cinco poliinsaturados (AGPI). De los AGS encontrados en mayor cantidad se presentó el palmítico $(23,64 \%)$, el esteárico $(9,43 \%)$ y el tricosanoico $(7,26 \%)$. De los AGMI los principales fueron el oleíco (22,63 $\%)$, el palmitoleico $(5,32 \%)$ y el vaccénico $(3,39 \%)$. Con respecto a los AGPI, los ácidos encontrados con mayor frecuencia fue el linoleico y el eicosatetraenoico (Cuadro 4).

Cuadro 4. Contenido de ácidos grasos saturados, insaturados y poliinsaturados de filetes de Hemiancistrus aspidolepis colectados en refugios mixtos de vida silvestre en Costa Rica. 2017.

Table 4. Saturated, unsaturated, and polyunsaturated fatty acid content of Hemiancistrus aspidolepis fillets sampled in Mixed Wildlife Refuges in Costa Rica. 2017.

\begin{tabular}{|c|c|c|c|c|}
\hline & Promedio & Máx & Mín & D.E \\
\hline Ácido graso saturado (AGS) & \multicolumn{4}{|c|}{$\%$ relativo con respecto a la grasa } \\
\hline Mirístico C14:0 & 1,53 & 2,88 & 0,39 & 0,82 \\
\hline Pentadecanoico C15:0 & 0,93 & 2,13 & 0,14 & 0,60 \\
\hline Palmítico C16:0 & 23,64 & 30,52 & 11,11 & 6,70 \\
\hline Margárico C17:0 & 0,95 & 1,37 & 0,21 & 0,47 \\
\hline Esteárico C18:0 & 9,43 & 12,77 & 3,84 & 2,84 \\
\hline Tricosanoico $(\mathrm{C} 23: 0)$ & 7,26 & 13,82 & 0,70 & 9,28 \\
\hline Araquidico C20:0 & 0,78 & 1,31 & 0,28 & 0,38 \\
\hline Laúrico C12:0 & 0,45 & 0,77 & 0,27 & 0,28 \\
\hline Behenico C22:0 & 0,88 & 2,47 & 0,34 & 1,06 \\
\hline Ácido graso monoinsaturado (AGMI) & \multicolumn{4}{|c|}{$\%$ relativo con respecto a la grasa } \\
\hline Miristoleico C14:1 & 0,37 & 0,72 & 0,02 & 0,49 \\
\hline Palmitoleico C16:1 & 5,32 & 11,56 & 0,42 & 3,94 \\
\hline Margaroleico C17:1 & 0,43 & 1,03 & 0,09 & 0,35 \\
\hline Oleico C18:1 ( $\omega-9)$ & 22,63 & 56,22 & 10,38 & 15,18 \\
\hline cis-11-eicosenico C20:1 ( $\omega-9)$ & 1,33 & 3,85 & 0,52 & 1,42 \\
\hline 8-11,14-eicosatrienoico C20:3 ( $\omega-9)$ & 0,93 & 1,85 & 0,10 & 0,64 \\
\hline Nervónico 24:1 ( $\omega-9)$ & 1,56 & 2,90 & 0,83 & 0,85 \\
\hline Vácenico C18:1 & 3,39 & 3,91 & 2,86 & 0,74 \\
\hline Petroselenico C18:1 & 0,21 & 0,32 & 0,09 & 0,12 \\
\hline Tras-11-vaccenico C18:1t & 0,18 & 0,26 & 0,11 & 0,08 \\
\hline Ácido graso poli-insaturado (AGPI) & \multicolumn{4}{|c|}{$\%$ relativo con respecto a la grasa } \\
\hline Linoleico C18:2 ( $\omega-6)$ & 19,21 & 30,33 & 7,75 & 8,02 \\
\hline$\alpha$-linolenico C18:3 ( $\omega-3)$ & 1,97 & 3,50 & 0,33 & 1,20 \\
\hline g- linolenico C18:3 ( $\omega-6)$ & 0,35 & 0,56 & 0,05 & 0,22 \\
\hline cis-11,14-eicosadienoico C20:2 ( $\omega$-6) & 0,30 & 0,51 & 0,14 & 0,14 \\
\hline cis-5,8,11,14,-eicosatetraenoico C20:4 ( $\omega-6)$ & 5,12 & 8,11 & 0,62 & 3,19 \\
\hline
\end{tabular}

Máx: máximo; Mín: mínimo; D.E: desviación estándar / Máx: maximum; Min: minimum; D.E.: standard deviation. 


\section{Contenido de mercurio}

Todas las muestras tuvieron niveles de mercurio total (Hg-T) inferior a $150 \mathrm{ng} \mathrm{g}^{-1}$ p.f. (que es igual a $0,15 \mathrm{ppm}$ ).

\section{Comparación del análisis proximal entre tipos de peces.}

Se compararon los análisis proximales de doce peces estudiadas incluyendo la especie H. aspidolepis de este estudio. De las anteriores la del pez diablo, la tilapia, trucha, pangasio y el pez gato son de aguas continentales, mientras que el pargo, la corvina, el lenguado y la barracuda son de aguas marinas.

Con respecto a la media general, H. aspidolepis mostró una diferencia superior de 3,8 g $100 \mathrm{~g}^{-1}$ (4,94 \%) para la

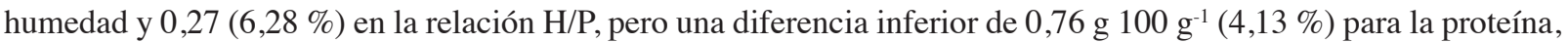

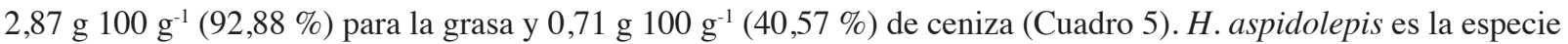
que tuvo la menor cantidad de grasa y ceniza entre los pescados analizados.

Cuadro 5. Comparación del análisis proximal de filetes de Hemiancistrus aspidolepis con resultados obtenidos de otras especies de peces consumidas por los seres humanos. Costa Rica, 2017.

Table 5. Comparison of the proximal analysis of Hemiancistrus aspidolepis fillets with obtained results for other fish species consumed by humans. Costa Rica, 2017.

\begin{tabular}{|c|c|c|c|c|c|c|}
\hline \multirow[b]{2}{*}{ Especie } & Humedad & Proteína & Grasa & Cenizas & $\mathbf{H} / \mathbf{P}^{*}$ & \multirow[b]{2}{*}{ Fuente } \\
\hline & \multicolumn{5}{|c|}{ g $100 \mathrm{~g}^{-1}$} & \\
\hline H. aspidolepis (pez diablo) & 80,69 & 17,64 & 0,22 & 1,04 & 4,57 & Este estudio \\
\hline Oreochromis sp. (tilapia) & 72,36 & 23,34 & 2,26 & 1,94 & 2,76 & Izquierdo-Córser et al. (2000) \\
\hline Oreochromis niloticus (tilapia) & 81,39 & 13,66 & 0,54 & 1,36 & 5,96 & Olapade et al. (2016) \\
\hline Oreochromis niloticus (tilapia) & 73,62 & 14,77 & 2,39 & 1,51 & 4,98 & Desta et al. (2019) \\
\hline Oncorhynchus mykiss (trucha) & 77,06 & 20,86 & 1,5 & 1,30 & 3,69 & Izquierdo-Córser et al. (2000) \\
\hline Pangasianodon hypophthalmus (pangasio) & 78,29 & 12,78 & 16,55 & 1,78 & 6,13 & Begum et al. (2012) \\
\hline Pangasianodon gigas (pez gato) & 78,88 & 19,00 & 0,54 & 1,47 & 4,15 & Chaijan et al. (2010) \\
\hline Lutjanus buccanella (pargo) & 76,72 & 20,99 & 1,12 & 1,33 & 3,66 & Izquierdo-Córser et al. (2000) \\
\hline Lutjanus guttatus (pargo) & 69,68 & 17,61 & 6,51 & 4,97 & 3,96 & Benitez-Hernández et al. (2017) \\
\hline Cysnoscion maracaiboensis (corvina) & 76,95 & 20,73 & 2,11 & 1,90 & 3,71 & Izquierdo-Córser et al. (2000) \\
\hline Cyclopsetta querna (lenguado) & 79,93 & 19,86 & 1,18 & 1,31 & 4,02 & $\begin{array}{c}\text { Fonseca-Rodríguez y Chavarría- } \\
\text { Solera (2017) }\end{array}$ \\
\hline Sphyraena ensis (barracuda) & 77,13 & 19,52 & 2,18 & 1,12 & 3,95 & $\begin{array}{l}\text { Fonseca-Rodríguez y Chavarría- } \\
\text { Solera (2017) }\end{array}$ \\
\hline Promedio & 76,89 & 18,40 & 3,09 & 1,75 & 4,30 & \\
\hline D.E & 3,48 & 3,23 & 4,54 & 1,05 & 0,98 & \\
\hline
\end{tabular}

*H/P: relación humedad/proteína; D.E: desviación estándar / *H/P: humidity/protein ratio; D.E.: standard deviation. No se presentaron diferencias estadísticas, $\mathrm{p}>0,05$ / There were no statistical differences, $\mathrm{p}>0.05$.

Con respecto al contenido de proteína en los peces de agua dulce, sobresale que $H$. aspidolepis estuvo por encima del promedio $(1,40 \%)$ y tuvo menos cantidad de grasa $(94,44 \%)$ y cenizas $(33,33 \%)$, comparado a los peces marinos, el pez diablo tuvo una mayor cantidad de humedad $(6,05 \%)$ y una menor cantidad de proteína $(10,64 \%)$, grasa $(91,57 \%)$ y cenizas $(51,09 \%)$, pero sin presentar diferencias estadísticas. 


\section{Discusión}

Respecto a las características físicas del $H$. aspidolepis, se aprecia que los individuos colectados no poseían tallas ni porcentaje de peso de filetes como los usuales de peces para mercado y consumo, ya que según Oliveira et al. (2015) un valor aceptable de rendimiento del tronco en peces marinos y de agua dulce debe ser entre $60 \mathrm{y}$ $63 \%$, de manera que los peces con mayor rendimiento de tronco pueden presentar mayor rendimiento de filete (Santaella et al., 2012), siendo lo anterior, más del doble del rendimiento obtenido en los peces de H. aspidolepis, que no superaron el $40 \%$ de rendimiento del filete.

El mayor porcentaje de peso estuvo representado por la carcasa, siendo esta hasta tres veces el peso de los filetes, lo cual se explica por el peso de las placas óseas que recubren el cuerpo de los loricaridos y el tamaño de la cabeza. Un estudio indica que las especies con cabeza grande pueden presentar un bajo rendimiento de la porción comestible en relación con el peso total, reportando datos de tamaños de cabeza que representaban hasta el 57\% de su peso corporal, como en el caso de Diapterus peruvianus (Carranza, 2018).

De los valores obtenidos del análisis proximal, el contenido de ácidos grasos y los minerales mostró que los filetes de la especie H. aspidolepis poseen un valor nutricional que permite su consumo por parte de los seres humanos.

Por las características propias de los peces, los filetes de pescado poseen altos contenidos de humedad, de hasta un $80 \%$ y, esto puede favorecer el crecimiento de bacterias, lo que podría generar un rápido deterioro del alimento si es almacenado de forma inadecuada (Izquierdo-Córser et al., 2000), sin embargo, H. aspidolepis presentó un valor cercano al recomendado por la Organización de las Naciones Unidas de la Alimentación y la Agricultura (Food and Agriculture Organization, 1999) para la relación humedad y lípidos, lo que favorecería la preservación del producto.

Con respecto a la proteína total, H. aspidolepis presentó un nivel cercano a valores de otras especies de importancia comercial (Food and Agriculture Organization, 2007) como la carne de res (22,3 g $\left.100 \mathrm{~g}^{-1}\right)$, pollo (22,8

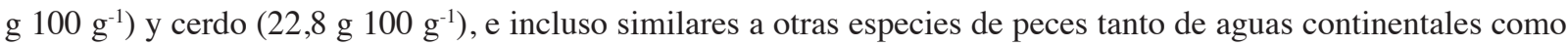
marinas, por lo que el consumo de esta especie es altamente recomendado por ser más nutritiva y tener un alto contenido de proteína total cercano a los estándares de mercado (Olapade et al., 2016). El Consejo de Alimentación y Nutrición de EE.UU., recomienda en individuos saludables adultos con actividad moderada una energía alimenticia de $3057 \mathrm{kcal} \mathrm{día}^{-1}$, por lo que el consumo filetes de $H$. aspidolepis puede contribuir significativamente a la dieta humana por su contenido de energía (Hernández, 2004).

El índice de jugosidad representado por la relación humedad/proteína fue superior al reportado por otras especies de peces que llegan a un promedio de 3,50, lo que indica que la carne fue jugosa y podría tener una mejor capacidad de aceptación sensorial por parte de las personas, incluso podría poseer características idóneas para la generación de subproductos, como por ejemplo los curados (Izquierdo-Córser et al., 2000).

Se presentó una relación inversa entre la grasa y la humedad, esto es concordante con los resultados obtenidos

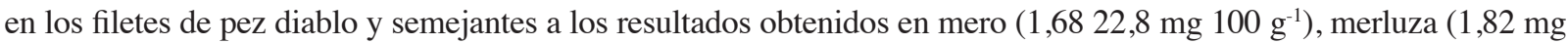
$\left.100 \mathrm{~g}^{-1}\right)$, robalo (1,66 mg $\left.100 \mathrm{~g} \mathrm{~g}^{-1}\right)$, trucha $\left(1,50 \mathrm{mg} 100 \mathrm{~g}^{-1}\right)$ y corvina $\left(2,11 \mathrm{mg} 100 \mathrm{~g} \mathrm{~g}^{-1}\right)$ (Izquierdo-Córser et al., 2000).

El contenido calcio $\left(28,28 \mathrm{mg} 100 \mathrm{~g}^{-1}\right)$ en $H$. aspidolepis fue superior a los presentados por otras especies cultivadas en aguas continentales como la tilapia $\left(15,66 \mathrm{mg} 100 \mathrm{~g}^{-1}\right)$ y la trucha $\left(10,78 \mathrm{mg} 100 \mathrm{~g}^{-1}\right)$ (IzquierdoCórser et al., 2000).

Los seres humanos necesitan de $18 \mathrm{mg} \mathrm{día}^{-1}$ de hierro, por lo que el consumo de $150 \mathrm{~g}$ de filete $(1,85 \mathrm{mg} \mathrm{Fe}$ $100 \mathrm{~g}^{-1}$ ) de H. aspidolepis brindaría un importe aporte complementario al requerimiento diario (Hernández, 2004). Con respecto al potasio y el sodio, se presentaron para Pangasius hypophthalmus valores de estos minerales de 335,6 y $387,5 \mathrm{mg} \mathrm{kg}^{-1}$, respectivamente (Orban et al., 2008), los cuales están por debajo de los obtenidos en $H$. aspidolepis. De igual manera, dado que los niveles promedio de $\mathrm{K}$ en $H$. aspidolepis estuvieron por encima del

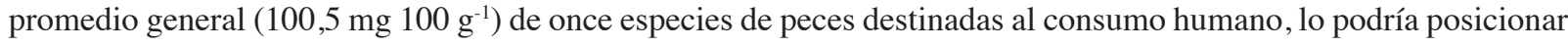
como una fuente importante de este elemento (Shehawy et al., 2016). 
El ácido palmítico fue el AGS en mayor cantidad, sin embargo, fue casi la mitad del mostrado en peces marinos como la merluza $(47,5 \%)$, el róbalo $(41,6 \%)$ y la lisa $(40,8 \%)$ y similar a la corvina, el pargo y la trucha (Izquierdo-Córser et al., 2000). Los AGMI más abundantes fueron el palmitoleico y el oleico en el estudio de

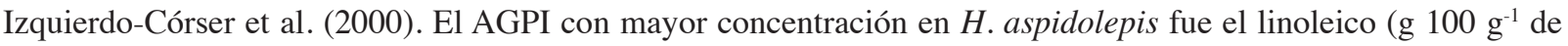
grasa), mismo que se encontró en alrededor de 47,4 g $100 \mathrm{~g}^{-1}$ de grasa en la tilapia (Izquierdo-Córser et al., 2000).

La ceniza encontrada en $H$. aspidolepis presentó valores cercanos a los de otras especies de peces tanto de aguas continentales como marinas, cercanos a 1,04 (g $100 \mathrm{~g}^{-1}$ ) (Fonseca-Rodríguez \& Chavarría-Solera, 2017; Olapade et al., 2016; Izquierdo-Córser et al., 2000). En cuanto al mercurio, la especie de estudio presentó un valor de $150 \mathrm{ng} \mathrm{g}^{-1}$ que está por debajo del indicado por la Agencia de Protección Ambiental (EPA) de los EEUU, quienes consideran como valor máximo para no representar riesgos para la salud humana, $0,46 \mu \mathrm{g} \mathrm{g}^{-1}$ por porción de pescado $(110 \mathrm{~g}$ de pescado crudo) a la semana (United States Environmental Protection Agency, 2018). Así también, en Canadá se utiliza un valor límite de $0,5 \mathrm{ppm}$ que es igual a $500 \mathrm{ng} \mathrm{g}^{-1}$ y la Organización Mundial de la Salud (OMS), establece

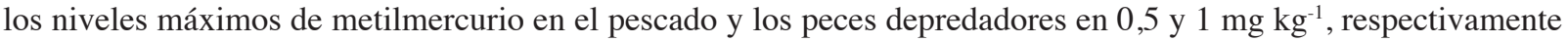
(Food and Agriculture Organization, \& World Health Organization, 2015), siendo estos límites superiores a los valores de mercurio obtenidos en el presente estudio. Una de las principales fuentes de ingreso de mercurio a los seres humanos es por medio del consumo de pescado, por su mayor capacidad de acumular este metal pesado (Ruiz, 2016), se esperaría que el consumo de H. aspidolepis sea seguro para el organismo humano, debido a que los niveles de mercurio son menores al máximo toxico para el ser humano.

\section{Conclusiones}

El contenido proximal, de ácidos grasos y minerales de H. aspidolepis fue similar o mejor para el consumo humano, que otras especies de peces tanto de aguas marinas como continentales, y los contenidos de proteínas y lípidos lo hace idóneo para su posible utilización y comercialización. Considerando estos valores, se podría concluir que hay un beneficio para el ser humano en el consumo de $H$. aspidolepis, aunque el rendimiento de los filetes no sea tan atractivo como otros peces comerciales.

Se puede reducir el impacto de esta especie invasora en las áreas naturales y protegidas a través de su consumo implementando alternativas de manejo.

\section{Agradecimientos}

Los autores agradecen a la Coordinación y miembros de la Unidad Técnica del Proyecto Humedales Programa de Naciones Unidas para el Desarrollo - Sistema Nacional de Áreas de Conservación 2014-2019. A los estudiantes de la carrera de Ingeniería en Acuicultura de la Universidad Técnica Nacional por el apoyo en el procesamiento de las muestras.

\section{Referencias}

Aguirre-Muñoz, A., Mendoza-Alfaro, R., Ponce-Bernal, H. A., Arriega-Cabrera, L., Campos-Gonzáles, E., Contretas-Balderas, S., Gutiérrez, M. E., Espinoza-García „F. L., Fernández-Salas, L., Galaviz-Silva, L., García de León, F. J., LazcanoVillareal, D., Martínez-Jiménez, M., Meave del Castillo, M. E., Medellín, R. A., Naranjo-García, E., Olivera-Carrasco, M. T., Pérez-Sandi, M., Rodríguez-Almaraz, G., Salgado Maldonado, G., et al. (2009). Especies exóticas invasoras: impactos sobre las poblaciones de flora y fauna, los procesos ecológicos y la economía. En R. Dirzo, R. González, \& I. 
J. March (Eds.), Capital natural de México (vol. II., pp. 277-318). Comisión Nacional para el Conocimiento y Uso de la Biodiversidad. https://www.biodiversidad.gob.mx/v_ingles/country/pdf/CapNatMex/Vol\%20II/II06_Especies\%20 exoticas\%20invasoras_Impactos\%20sobre\%20las\%20pobla.pdf

Angulo, A., Garita-Alvarado, C., Bussing, W., \& López, M. (2013). Annotated checklist of the freshwater fishes of continental and insular Costa Rica: additions and nomenclatural revisions. Check List, 9(5), 987-1019. https://doi. $\operatorname{org} / 10.15560 / 9.5 .987$

Association of Official Analytical Chemists. (2019). Official methods of analysis of AOAC International (21st ed.). Vol. 1. http:// www.eoma.aoac.org/

Begum, M., Akhter, T., \& Minar, M. H. (2012). Analysis of the proximate composition of domesticated pangus (Pangasius hypophthalmus) in laboratory condition. Journal of Environmental Science and Natural Resources, 5(1), 69-74. https://doi.org/10.3329/jesnr.v5i1.11555

Benitez-Hernández, A., Hernández, C., Ibarra-Castro, L., Sánchez-Gutiérrez, Y., \& Gaxiola, G. (2017). Efecto de diferentes niveles de proteína y carbohidratos en juveniles de pargo flamenco Lutjanus guttatus sobre la composición proximal y parámetros hematológicos. World Aquaculture Society. Obtenido 14 jul. 2009 de https://www.was.org/meetings/ ShowAbstract .aspx ?Id=52379.

Blackburn, T. M., Pysek, P., Bacher, S., Carlton, J., Duncan, R., Jarosik, V., Wilson, J., \& Richardson, D. (2011). A proposed unified framework for biological invasions. Trends in Ecology \& Evolution, 26(7), 333-339. https://doi.org/10.1016/j. tree.2011.03.023

Bussing, W. (2002). Peces de aguas continentales de Costa Rica (2da ed.). Editorial de la Universidad de Costa Rica.

Carranza, E. (2018). Rendimiento corporal de especies de peces nativos del Golfo de Fonseca de Honduras. Revista Ciencia y Tecnología, 23,46-59. https://doi.org/10.5377/rct.v0i23.6860

Carrillo, L., Dalmau, J., Román, J., Solà, R., \& Pérez, F. (2011). Grasas de la dieta y salud cardiovascular. Atención Primaria, 43(3), 157.e1-157.e16. https://doi.org/10.1016/j.aprim.2010.12.003

Castillo, D. (2018). Abundancia, relaciones longitud-peso y aspectos reproductivos de la tilapia (Oreochromis niloticus) y del pleco (Hypostomus cf. aspidolepis) en el Río Sabogal, cuenca del Río Frío, vertiente atlántica norte de Costa Rica [Tesis Lic. Universidad de Costa Rica]. Repositorio SIBDI-UCR. http://repositorio.sibdi.ucr.ac.cr:8080/jspui/ handle/123456789/6434

Castro, M. I., Maafs, A. G., \& Galindo, C. (2013). Perfil de ácidos grasos de diversas especies de pescados consumidos en México. Revista de Biología Tropical, 61(4), 1981-1998.

Chaijan, M., Jongjareonrak, A., Phatcharat, S., Benjakul, S., \& Rawdkuen, S. (2010). Chemical compositions and characteristics of farm raised giant catfish (Pangasianodon gigas) muscle. Food Science Technology, 43(3),452-457. https://doi. org/10.1016/j.lwt.2009.09.012

Covain, R., \& Fisch-Muller, S. (2007). The genera of the Neotropical armored catfish subfamily Loricariinae (Siluriformes: Loricariidae): a practical key and synopsis. Zootaxa, 1462, 1-40. https://doi.org/10.11646/zootaxa.1462.1.1

Deines, A. M. (2013). Environmental change and tradeoffs in freshwater ecosystem services: Nile Tilapia (Oreochromis niloticus) introduction to the Kafue River, Zambia [Ph.D. Diss., University of Notre Dame]. https://curate.nd.edu/ show/s4655d8934f 
Desta, D. T., Zello, G., Alemayehu, F. R., Estfanos, T. K., Zatti, K., \& Drew, M. (2019). Proximate analysis of Nile Tilapia, (Oreochromis niloticus), Fish fillet harvested from farmers pond and Lake Hawassa, Southern Ethiopia. International Journal for Research and Development in Technology, 11(1), 94-99.

Food and Agriculture Organization. (1999). El Pescado Fresco: su calidad y cambios de su calidad. http://www.fao.org/3/ v7180s/v7180s00.htm\#Contents

Food and Agriculture Organization. (2007). Meat processing technology for small- to medium-scale producers. http://www.fao. org/ag/againfo/themes/es/meat/backgr_composition.html

Food and Agricultural Organization, \& World Health Organization. (2015). Codex Alimentaruis: Norma General para los contaminantes y las toxinas presentes en los alimentos y piensos. http://www.fao.org/input/download/standards/17/ CXS_193s_2015.pdf

Fonseca, R., \& Vargas, P. (2018). Estudio de factibilidad del aprovechamiento económico de una especie invasora Hypostomus plecostomus en el humedal de Caño Negro, Costa Rica. Journal of Marine and Coastal Science, 10(2), 31-49. https:// doi.org/10.15359/revmar.10-2.2

Fonseca-Rodríguez, C., \& Chavarría-Solera, F. (2017). Composición proximal en algunas especies de pescado y mariscos disponibles en el pacífico costarricense. Uniciencia, 31(1), 23-28. https://doi.org/10.15359/ru.31-1.3

Gibbs, M. A., Shields, J. H., Lock, D. W., Talmadge, K. M., \& Farrell, T. M. (2008). Reproduction in an invasive exotic catfish Pterygoplichthys disjunctivus in Volusia Blue Spring, Florida, USA. Journal of Fish Biology, 73(7), 1562-1572. https://doi.org/10.1111/j.1095-8649.2008.02031.x

Guerrero, T. (2016). Propuesta base para la estrategia de manejo y control local del pez diablo en el Refugio Nacional de Vida Silvestre mixto Caño Negro, Costa Rica [Tesis MSc., CATIE]. Repositorio CATIE. http://hdl.handle.net/11554/8604

Hernández, M. (2004). Recomendaciones nutricionales para el ser humano. Revista Cubana de Investigaciones Biomédicas, 23(4), 266-292.

Hoover, J. J., Murphy, C. E., \& Killgore, J. (2014). Ecological impacts of suckermouth catfishes (Loricariidae) in North America: a conceptual model. ANSRP Bulletin, 14(1), 1-20.

Howard, G. (1999). Especies invasoras y humedales. Ramsar. https://www.ramsar.org/sites/default/files/documents/cop7-docs/ NON-RESRECS\%20FINAL/COP7\%2024S.pdf

Izquierdo-Córser, P., Torres-Ferrari, G., Barbosa-de-Martínez, Y., Márquez-Salas, E., \& Allara-Cagnasso, M. (2000). Análisis proximal, perfil de ácidos grasos, aminoácidos esenciales y contenido de minerales en doce especies de pescado de importancia comercial en Venezuela. Archivos Latinoamericanos de Nutrición, 50(2),187-194.

Njinkoue, J. M., Gouado, I., Tchoumbougnang, F., Ngueguim, J. H. Y., Ndinteh, D. T., Fomogne-Fodjo, C. Y., \& Schweigert, F. J. (2016). Proximate composition, mineral content and fatty acid profile of two marine fishes from Cameroonian coast: Pseudotolithus typus (Bleeker, 1863) and Pseudotolithus elongatus (Bowdich, 1825). NFS Journal, 4, 27-31. https://doi.org/10.1016/j.nfs.2016.07.002

Nico, L. G., Jelks, H. L., \& Tuten, T. (2009). Non-native suckermouth armored catfishes in Florida: description of nest borrows and burrow colonies with assessment of shoreline conditions. ANSRP Bulletin, 9(1), 1-30.

Orban, E., Nevigato, T., Lena, G. D., Masci, M., Casini, I., Gambelli, L., \& Caproni, R. (2008). New trends in the seafood market. Sutchi catfish (Pangasius hypophthalmus) fillets from Vietnam: Nutritional quality and safety aspects. Food Chemistry, 110(2), 383-389. https://doi.org/10.1016/j.foodchem.2008.02.014 
Oliveira, V. D., Vieira, C., Dessinoni, M., Pimentel, R., Fonseca, R., \& Solis, L. (2015). Using morphometric variables in evaluations of body of fish yields. Journal of Veterinary Medicine and Research, 2(4), 1032-1036.

Olapade, O. A., Taiwo, I. O., Lamidi, A. A., \& Awonaike, O. A. (2016). Proximate composition of Nile Tilapia (Oreochromis niloticus) and Tilapia Hybrid (Red Tilapia) from Oyan Lake, Nigeria. Bulletin of University of Agricultural Science and Veterinary Medicine Food Science and Technology, 73(1), 19-23. https://doi.org/10.15835/buasvmcn-fst:11973

Proyecto Humedales de Sistema Nacional de Áreas de Conservación - Programa de Naciones Unidad para el Desarrollo Global Enviromental Facility. (2018). Ecosistemas Vegetales del Complejo de Humedales de Caño Negro, Los Chiles, Costa Rica. https://labmeh.catie.ac.cr/wp-content/uploads/2018/06/Ecosistemas-de-Ca\%C3\%B1o-Negro_2018-1.pdf

Ruíz, I. (2016). Metodologías analíticas utilizadas actualmente para la determinación de mercurio en músculo de pescado. Revista Pensamiento Actual, 16(26), 113-122. https://doi.org/10.15517/PA.V16I26.25187

Santaella, M., Graciá, C. M., Peragio, M. J., \& Santaella, J. (2012). Evaluación sensorial de diferentes presentaciones comerciales de dorada (Sparus aurata) de acuicultura. Anales de Veterinaria de Murcia, 28, 85-96. https://doi. org/10.6018/j/188751

Shehawy, S. M. E., Gab-Alla, A. A., \& Mutwally, H. M. A. (2016). Proximate and elemental composition of important fish species in Makkah central fish market, Saudi Arabia. Food and Nutrition Science, 7(6), 429-439. https://doi. org/10.4236/fns.2016.76044

United States Environmental Protection Agency. (2018). EPA-FDA Fish Advice: Technical Information. United States Environmental Agency. https://www.epa.gov/fish-tech/epa-fda-fish-advice-technical-information

Undeland, I., Ellegard, L., \& Sandberg, A. S. (2004). Fish and cardiovascular health. Scandinavian Journal of Nutrition, 48(3),119-130. https://doi.org/10.1080/11026480410000427

Varlık, C., Erkan, N., \& Baygar, T. (2004). Su Ürünleri besin bileşimi. In: C. Varlık, (Ed.), Su Ürünleri Isşleme Teknolojisi (Vol. 1, pp. 1-45). Istanbul Üniversitesi Yayın. 